\title{
The predictive role of small airway dysfunction and airway inflammation biomarkers for asthma in preschool and school-age children: a study protocol for a prospective cohort study
}

\author{
Qinyuan $\mathrm{Li}^{1}{ }^{1}$, Qi Zhou ${ }^{2}$, Yuanyuan $\mathrm{Li}^{1}$, Enmei Liu ${ }^{1}$, Zhou Fu ${ }^{1}$, Jian Luo ${ }^{1}$, Sha Liu ${ }^{1}$, Fangjun Liu ${ }^{1}$, \\ Yaolong Chen ${ }^{2,3,4}$, Zhengxiu Luo ${ }^{1}$ \\ ${ }^{1}$ Department of Respiratory Medicine, Children's Hospital of Chongqing Medical University, National Clinical Research Center for Child Health \\ and Disorders, Ministry of Education Key Laboratory of Child Development and Disorders, Chongqing Key Laboratory of Pediatrics, Chongqing, \\ China; ${ }^{2}$ Evidence-based Medicine Center, School of Basic Medical Sciences, Lanzhou University, Lanzhou, China; ${ }^{3}$ Lanzhou University Institute of \\ Health Data Science, Lanzhou, China; ${ }^{4}$ WHO Collaborating Centre for Guideline Implementation and Knowledge Translation, Lanzhou, China \\ Contributions: (I) Conception and design: Z Luo, Q Li, Q Zhou; (II) Administrative support: Z Luo, E Liu, Z Fu, Y Chen; (III) Provision of \\ study materials or patients: Q Li, J Luo, Z Luo, E Liu, Z Fu; (IV) Collection and assembly of data: Q Li, Q Zhou, Y Li; (V) Data analysis and \\ interpretation: Q Li, Z Luo, S Liu, F Liu; (VI) Manuscript writing: All authors; (VII) Final approval of manuscript: All authors. \\ Correspondence to: Zhengxiu Luo, MD. Children's Hospital of Chongqing Medical University, No. 20 Jinyu Avenue, Liangjiang New District, \\ Chongqing 401122, China. Email: luozhengxiu816@163.com.
}

Background: Preschool children are at a high risk of developing asthma. Asthma in preschool children could remit in most cases, but could persist into school age, adolescence, or even adulthood in some cases. However, it is difficult to predict which children with preschool asthma will develop into school-age asthma. We present a cohort study protocol to explore the predictive role of small airway dysfunction and airway inflammation biomarkers of asthma in preschool and school-age children.

Methods: A prospective cohort study will be conducted with at least 205 children with preschool asthma. All patients will be recruited when they consult a pediatric pulmonologist at the Children's Hospital of Chongqing Medical University and will be followed up to 6 years of age. Initially, patients' demographic information, medical history, physical findings, and questionnaire information will be collected, and baseline small airway function and inflammation biomarkers will be detected. During the follow-up period, medical history, physical findings, and the questionnaire results will be collected every 3 months, and small airway function will be tested by impulse oscillometry (IOS) every 6 months. At the final visit, a definite diagnosis of school-age asthma will be made by a pediatric pulmonologist based on the criteria of the Global Initiative for Asthma 2020.

Discussion: The study will be the first to be conducted in preschool children assessing whether small airway dysfunction combined with airway eosinophilic biomarkers and club cell secretory protein is associated with school-age asthma. This study may provide new promising predictors of persistent asthma from preschool to school age.

Trial Registration: The study has been registered at the Chinese Clinical Trial Registry (ChiCTR2000039583). Registered on November 1, 2020. Protocol version: version 1.0, August 16, 2021.

Keywords: Pediatric asthma; preschool; school age; small airway dysfunction (SAD); club cell secretory protein (CC16)

Submitted May 27, 2021. Accepted for publication Sep 06, 2021.

doi: $10.21037 / \mathrm{tp}-21-239$

View this article at: https://dx.doi.org/10.21037/tp-21-239

^ ORCID: 0000-0002-4052-5747. 


\section{Introduction}

Pediatric asthma remains an important health concern, as its prevalence remains high despite current treatment (1). Preschool children are at a high risk for asthma (2). Although most patients can recover, some develop persistent asthma into school age, adolescence, and even adulthood $(3,4)$. Early identification and treatment are associated with improved asthma control and a reduced risk of developing asthma at school age (5). Therefore, it is crucial to investigate the predictors of persistent asthma from preschool to school age.

Several systematic reviews have evaluated predictors of the development of asthma at school age (6-8). The male sex, exposure to smoke, family history of asthma or allergy, allergic disease comorbidities or precursors of asthma (eczema, allergic rhinitis, or respiratory infections), severity of early symptoms (frequency of wheeze, wheeze without colds, dyspnea, or obstruction) and specific IgE to inhalant or food allergens, and polysensitisation (allergy to more than one allergen) were predictors of developing asthma at school age (6-8). However, the correlations between these factors and school-age asthma were weak. Moreover, measurement of the majority of these factors was subjective (6-8). Therefore, new reliable predictors of asthma development at school age need to be identified.

Abnormal airway function is the main characteristic of asthma (9). Assessment of airway function is essential for understanding disease progression in early childhood and may predict asthma in later life (10-12). However, most studies have focused on the functions of large airways. The role of small airway function in asthma control has gained attention recently $(13,14)$. Small airways are defined as distal airways with diameters of $<2 \mathrm{~mm}(13,14)$. The intensity of inflammation was higher in the small airways than in the large airways in patients with asthma (15). Small airway remodeling occurs during at the early onset of asthma (16). Small airway dysfunction (SAD), determined by impulse oscillometry (IOS) in preschool children with asthma has been shown to predict impaired lung function by spirometry in the adolescent (17). Additionally, some studies have indicated that SAD is strongly correlated with uncontrolled asthma $(13,18)$. Therefore, SAD may be a promising early predictor of poor prognosis in preschool asthma (19).

Airway inflammation is particularly important for the development of SAD and asthma (9). Excessive inflammation leads to airway epithelial injury, abnormal repair, and inappropriate remodeling, accelerating the decline in small airway function and recurrent attacks in patients with asthma (9). The measurement of airway inflammation may be helpful in understanding asthma progression. The fraction of exhaled nitric oxide (FeNO) and blood eosinophils are biomarkers of eosinophilic airway inflammation (20). A study has reported that increased FeNO levels in infants and toddlers had a higher risk of asthma and increased airway reactivity at 5 years of age (21). An increased eosinophil count in preschoolers increases the risk of uncontrolled asthma at school age (22). In addition to biomarkers of eosinophilic airway inflammation, biomarkers of lung epithelial injury may also be potential predictors for poor asthma prognosis. Club cell secretory protein $(\mathrm{CC} 16)$ is an anti-inflammatory protein secreted by the club cells in the distal airways and can be measured in circulation (23). Lower CC16 levels in the blood and airways have been proposed as biomarkers of lung epithelial injury and may predict poor prognosis of asthma $(23,24)$.

Studies have shown that SAD and abnormal inflammation biomarkers in preschool children are associated with poor asthma control and increased hyperresponsiveness in later life. However, whether they are associated with developing asthma at school age remains unclear. Moreover, evidence has shown that a combination of SAD and inflammation biomarkers may have a better predictive performance than either biomarker alone (25).

The cohort study will aim to investigate whether SAD, airway inflammation biomarkers, or a combination of SAD and airway inflammation biomarkers are associated with asthma at school age. We will also further investigate whether combined biomarkers enhance reliable school-age asthma prediction. Finally, we will investigate whether SAD and abnormal inflammation biomarkers are associated with the frequency of exacerbations and asthma control levels. Although a few studies have investigated their associations $(18,21,22)$, these results require further validation.

\section{Study aims}

To investigate whether SAD, airway inflammation biomarkers, or a combination of SAD and airway inflammation biomarkers are associated with asthma at school age.

\section{Methods and analysis}

\section{Study design and setting}

This prospective cohort study will follow-up children with asthma from preschool to 6 years of age. Participants will 


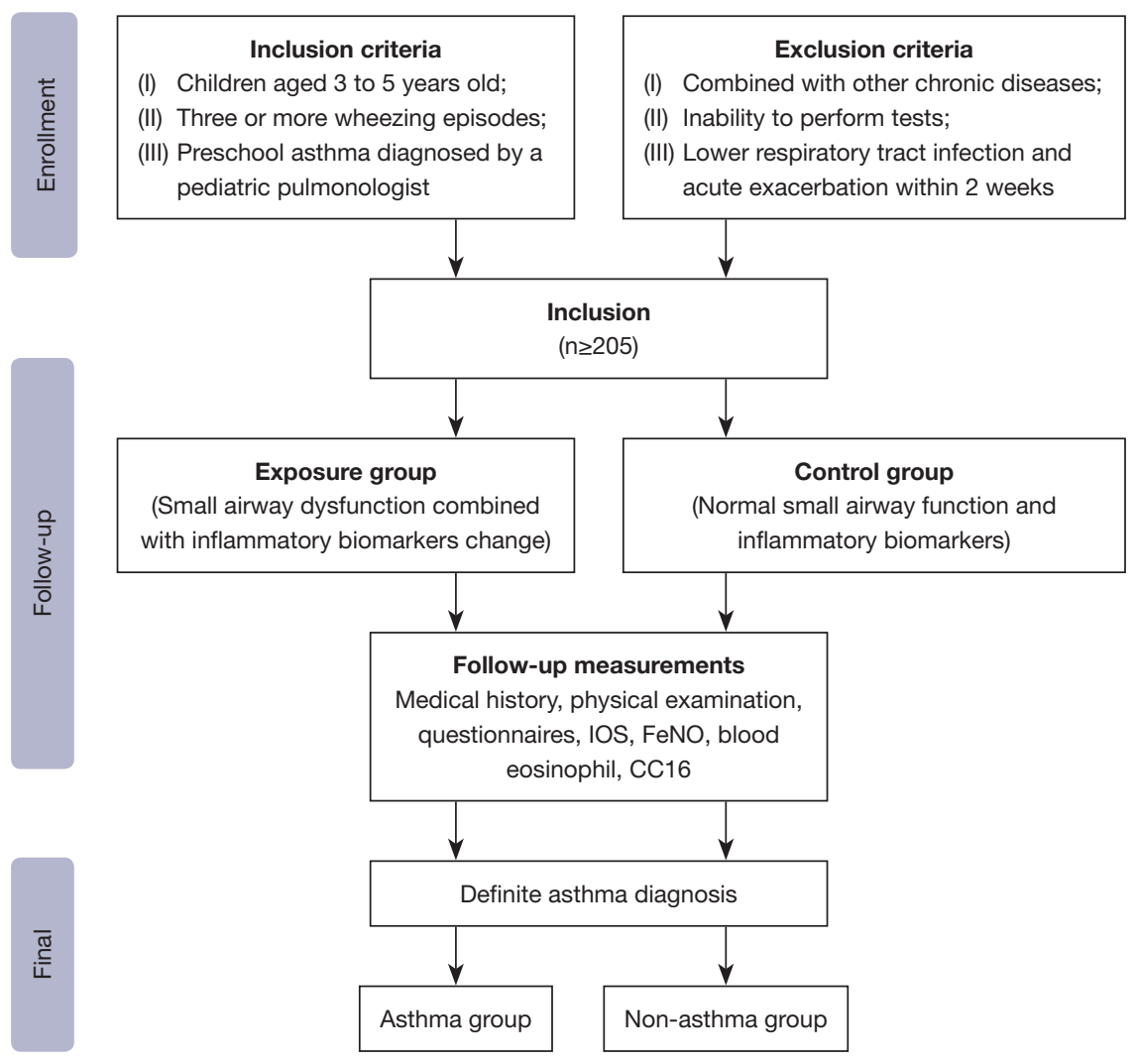

Figure 1 Summary of study procedures. IOS, Impulse oscillometry; FeNO, fraction of exhaled nitric oxide; CC16, club cell secretory protein.

be recruited from the Children's Hospital of Chongqing Medical University, a 2,000-bed tertiary teaching hospital in Chongqing, China, ranked in the top three of all domestic children's hospitals.

\section{Eligibility criteria}

\section{Inclusion criteria}

(I) Children aged 3 to 5 years old;

(II) Three or more wheezing episodes;

(III) Asthma diagnosed by a pediatric pulmonologist according to the criteria established by Canadian Thoracic Society and Canadian Pediatric Society (26).

\section{Exclusion criteria}

(I) Mental retardation, cardiac anomalies, congenital malformations, other diseases of the lungs/airways, and Crohn's disease or rheumatic arthritis;

(II) The inability to perform lung function tests or inflammatory biomarker measurements;
(III) A history of lower respiratory tract infection and acute exacerbation within 2 weeks before the tests.

\section{Outcomes}

The primary outcome will be the development of asthma at school age. It will be estimated by a pediatric pulmonologist following the criteria of the Global Initiative for Asthma (GINA) 2020 (27) at the final visit. The secondary outcomes will be the frequency of exacerbations and asthma control assessed by pediatric respiratory physicians using the Childhood Asthma Control Test (C-ACT) $(28,29)$ or the Test for Respiratory and Asthma Control in Kids (TRACK) $(30,31)$ at every visit.

\section{Study procedures}

A summary of the study procedure is presented in Figure 1. The recruitment start date will be Oct 1, 2021. The anticipated recruitment end date will be Dec 1, 2022 and 
Table 1 Overview of measurements per visit

\begin{tabular}{|c|c|c|c|c|c|c|c|c|c|c|c|c|}
\hline Measures & $\frac{\text { Enrollment }}{\mathrm{V} 1}$ & \multicolumn{10}{|c|}{ Follow-up } & $\frac{\text { Final }}{\text { V12 }}$ \\
\hline Eligibility screen & $\sqrt{ }$ & & & & & & & & & & & \\
\hline Informed consent & $\sqrt{ }$ & & & & & & & & & & & \\
\hline Demographic information & $\sqrt{ }$ & & & & & & & & & & & \\
\hline Physical examination & $\sqrt{ }$ & $\sqrt{ }$ & $\sqrt{ }$ & $\sqrt{ }$ & $\sqrt{ }$ & $\sqrt{ }$ & $\sqrt{ }$ & $\sqrt{ }$ & $\sqrt{ }$ & $\sqrt{ }$ & $\sqrt{ }$ & $\sqrt{ }$ \\
\hline Asthma control questionnaires & $\sqrt{ }$ & $\sqrt{ }$ & $\sqrt{ }$ & $\sqrt{ }$ & $\sqrt{ }$ & $\sqrt{ }$ & $\sqrt{ }$ & $\sqrt{ }$ & $\sqrt{ }$ & $\sqrt{ }$ & $\sqrt{ }$ & $\sqrt{ }$ \\
\hline IOS & $\sqrt{ }$ & & $\sqrt{ }$ & & $\sqrt{ }$ & & $\sqrt{ }$ & & $\sqrt{ }$ & & $\sqrt{ }$ & $\sqrt{ }$ \\
\hline Spirometry & & & & & & & & & & & & $\sqrt{ }$ \\
\hline CC16 & $\sqrt{ }$ & & & & & & & & & & & $\sqrt{ }$ \\
\hline
\end{tabular}

IOS, impulse oscillometry; FeNO, fraction of exhaled nitric oxide; CC16, club cell secretory protein.

could be extended if the number of participants does not reach the expected sample size or a force majeure event impacts on the recruitment process. At the initial visit, the investigators will recruit potential participants based on the eligibility criteria and ask them to sign the consent form. For consenting participants, their demographic information and medical history will be obtained, including but not limited to sex, exposure to smoke, family history of asthma or allergy, and allergic disease comorbidities or precursors of asthma (eczema, allergic rhinitis, or respiratory infections). A brief physical examination will be performed, asthma control level will be assessed using the C-ACT or TRACK questionnaires (28-31), and IOS tests and inflammatory biomarker measurements will be performed. During the follow-up period, medical history, physical findings, and questionnaire results will be collected every 3 months, and the IOS test will be performed every 6 months. At the final visit, spirometry, a bronchodilation test, or a challenge test will be conducted; a definite diagnosis of asthma will be made following the criteria in GINA 2020 (27). All biological specimens will be stored in a locked freezer using identification codes. Table 1 provides a summary of the data to be collected.

\section{Sample size}

The following equation will be used to calculate the sample size of this study:

$$
n=\frac{\left(z_{a} \sqrt{2 p q}+z_{\beta} \sqrt{p_{0} q_{0}+p_{1} q_{1}}\right)^{2}}{\left(p_{1}-p_{0}\right)^{2}}
$$

$p_{0}$ : the percentage of preschool children with asthmatic symptoms and normal lung function who will develop asthma in adolescence; $p_{1}$ : the percentage of preschool children with asthmatic symptoms and SAD who will develop asthma in adolescence, $p=\left(p_{0}+p_{1}\right) / 2, q_{0}=1-\mathrm{p}_{0}, q_{1}$ $=1-p_{1}, q=1-p$.

Based on a previous study (32), $8 \%$ of children with normal lung function will develop severe persistent asthma in later life $\left(p_{0}=0.08\right)$, and $27 \%$ of children with SAD will develop severe persistent asthma in later life $\left(p_{1}=0.27\right)$. Assuming $\alpha=0.05$ (two-tailed), $\beta=0.1, Z_{\alpha}=1.96, Z_{\beta}=1.282$, drop-out rate $=20 \%$, and the number of children with normal lung function $=$ the number of children with $\mathrm{SAD}$, the total number of the participants would be 205 . We planned to recruit at least 205 patients for this study.

\section{Data collection methods}

\section{Lung function tests}

Participants will be asked to stop the use of shortacting beta-agonists and inhaled corticosteroids $(4 \mathrm{~h})$, anticholinergics, leukotriene modifiers, and long-acting beta-agonists $(24 \mathrm{~h})$ before the measurement.

During the follow-up period, IOS (Masterscreen IOS/ 
Sentry Suite, VyAire Medical) will be performed in triplicate according to the European Respiratory Society (ERS) guidelines $(33,34)$. During measurements, the children will be in a sitting position and breath through a mouthpiece using a nose clip. To minimize pressure losses in the IOS, the investigator will support the cheek. Respiratory resistance at 5 and $20 \mathrm{~Hz}$ (R5 and R20) will be used as indices of total and large airway resistance, respectively. The difference between R5 and R20 (R5-R20) will reflect the resistance of the small airways. Moreover, the following variables will be collected: reactance at $5 \mathrm{~Hz}(\mathrm{X} 5)$ reflecting the elastic properties of the small airways and resonant frequency (Fres), determined as the frequency at which the reactance equals zero $(34,35)$. The IOS parameters of R5, R20, R5-20, Fres, and X5 will be expressed as z-scores of height- and sex-corrected reference values (36). SAD will be defined by the exceedance of the normal reference curve 1.645 standard deviation (SD) threshold ( $Z$ scores $\pm 1.645 \mathrm{SD})(18)$.

At the final visit, spirometry (Masterscreen Paediatric PFT) will be conducted in accordance with the ERS guidelines (37). Forced mid-expiratory flow between $25 \%$ and $75 \%$ of forced vital capacity $\left(\mathrm{FEF}_{25-75}\right)$ will be measured to evaluate the resistance of small airways. Reference values for spirometry in children, adjusted for height and sex, will be applied (36). $\mathrm{FEF}_{25-75}<65 \%$ of the predicted values will be indicative of abnormal results. After the baseline measurements, a bronchodilation test or an outdoor exercise/methacholine challenge test will be conducted.

\section{Inflammatory biomarkers}

FeNO (Exhaled Breath Analyzer, Aerocrine AB, Stockholm, Sweden) will be performed in duplicate at a standard flow rate of $50 \mathrm{~mL} / \mathrm{s}$ for at least $6 \mathrm{~s}$, according to ERS/American Thoracic Society guidelines (38). The mean values of these measurements will be recorded as the final result. The upper limit of normal for FeNO level is considered $23.0 \mathrm{ppb}$ (39).

Peripheral blood eosinophil counts will be obtained from the standard complete blood counts. Eosinophilia will be defined as a peripheral blood eosinophil count of $\geq 0.4 \times 10^{9} / \mathrm{L}$ and eosinophils comprising $\geq 4 \%$ of leukocytes (40). CC16 levels will be measured in serum and/or induced sputum using a commercially available ELISA kit according to the manufacturer's instructions (Sangon Biotech, China).

\section{Asthma control questionnaires}

C-ACT is applicable to children aged 4-11 years (28). The questionnaire includes 7 questions. Questions 1-4 will be completed by children on current asthma symptoms, limitation of physical activities, and symptoms at night (each scored from 0 to 3). Questions 5-7 will be completed by the caregivers on day and night wheezing and other symptoms (each with scores ranging from 0 to 5). The total C-ACT score ranges from 0 to 27 . A higher score indicates better asthma control. Less than 20 points indicate uncontrolled asthma (29).

TRACK will be used in children $<5$ years of age (30). TRACK is a 5 -item standardized questionnaire, with four questions that address the impairment domain and one question that addresses the risk domain of asthma control. Each item is scored from 0 to 20 points on a 5 -point Likerttype scale. The total TRACK score ranges from 0 to 100 . A higher score indicates better asthma control. A score of $<80$ points suggests uncontrolled asthma (33).

\section{Study withdrawal}

Every effort should be made for patient follow up during the study period, including communicating with the patients through regular visits, telephone calls or social media such as WeChat. Following enrollment, participants may withdraw from follow-up in the study at any time at their request. If any participant withdraws from the study, the data before withdrawal will be included in the analysis. The participant will be required to complete the withdrawal visit in the case report form. The reasons for withdrawal will be presented in a summary table.

\section{Data management}

The collected data will be entered into the database by two researchers and will be checked and cleaned by clinicians and statisticians. All data will be labeled with participant identification codes, uploaded and stored in the Research Electronic Data Capture, an online data collection platform (41).

\section{Statistical analysis}

Simple descriptive statistics will be used to describe the enrolled patients. Categorical data will be summarized as numbers and frequencies. Continuous variables will be presented as mean $\pm \mathrm{SD}$ or median and interquartile range (IQR). Differences in the proportions of SAD between school-age children with and without asthma will be compared using a two-sided $\chi^{2}$ test or the Fisher exact tests. 
Differences in means or medians of FeNO, blood eosinophil count, and CC16 between school-age children with and without asthma will be compared using two-sided $t$-tests or analysis of variance comparisons. We will use a multivariate logistic regression model to obtain the risk ratios (RR) and their $95 \%$ confidence intervals for developing asthma at school age and adjusted them for the following potential confounders: sex, exposure to smoke, family history of asthma or allergy, and allergic disease comorbidities or precursors of asthma. The analysis strategy outlined for the primary outcome will be used as the secondary outcomes. We will use a single imputation or multiple imputation to handle missing data according to the guidelines (42). Statistical significance will be defined as a two-tailed $\mathrm{P}$ value $<0.05$. Data analyses will be performed using the SPSS software for Windows, v.22 (SPSS Inc., Chicago, IL, USA).

\section{Data monitoring}

The observational study will be conducted in the direction of the data monitoring committee (DMC). The DMC will be independent of the sponsor and competing interests. On a regular basis, the DMC will review the following, but not exhaustively: patient informed consent, patient recruitment and follow-up, adverse event documentation and reporting, statistics, quality of data, and investigator and patient compliance with protocol requirements.

\section{Risks of study participation}

There are minimal risks associated with this study. The risks of having blood taken include transient pain, bleeding, bruising, fainting, and rarely, infection or a blood clot where the needle enters the body. If any study-related adverse events occur, the investigator will report the event according to the requirements of the ethics board of the Children's Hospital of Chongqing Medical University.

\section{Ethical approval}

The study will be conducted in accordance with the Declaration of Helsinki (as revised in 2013). The study was approved by ethics board of the Chongqing Medical University Affiliated Children's Hospital (No.: 2020-217) and informed consent will be taken from all individual participants once the study is initiated. All participants will be informed of the fullest extent of the study in terms of understanding. A copy of the signed and dated written informed consent form will be provided to the participant. The confidentiality of all study participants will be protected in accordance with national regulations and only investigators will have access to the data.

\section{Dissemination of the study results}

We will disseminate the results through multiple ways such as publication in journals, oral presentations at multiple conferences, use professional writers to write the reports, and share all the relevant materials related to the studies through social media.

\section{Discussion}

Identifying the predictors of persistent asthma from preschool to school age is essential for preventing asthma progression. Pediatricians aim to find some reliable predictors, however, few clinical indexes have a good predictive value (6-8). The airflow limitation and airway inflammation are the main pathological hallmarks of asthma. It is reasonable to assume that the objective markers reflecting airway function and airway inflammation may have the potential to become reliable predictors of asthma progression. This study will be the first cohort study to assess whether SAD combined with airway eosinophilic biomarkers and CC16 is associated with school-age asthma in preschool children. In addition, we will use IOS instead of spirometry to detect the small airway function of preschool children with asthma. Spirometry is a traditional method to detect airways function (43). However, preschool children are difficult to complete spirometry due to poor cooperation. IOS requires minimal patient cooperation and is particularly useful to detect small airway changes (44). The use of IOS will improve the test accuracy of SAD.

\section{Study registration}

This study has been registered at the Chinese Clinical Trial Registry (ChiCTR2000039583) on Nov 1, 2020. We registered study objectives, eligibility criteria of participants, study design and setting, outcomes, data collection and management, and ethical approval.

\section{Acknowledgments}

We gratefully acknowledge the contribution of children and parents who participated in this study. We thank the 
evidence-based medicine center of Lanzhou University for assistance in the design of the study and statistical advice. We thank Children's Hospital of Chongqing Medical University for the provision of study settings and materials. Funding: The National Clinical Research Center for Child Health and Disorders will fund this study in patient recruitment, collection, management, and analysis of data, writing and publishing of the report, dissemination of the study results. The funder will not have ultimate authority over any of these activities. The funding number is NCRCCHD-2020-GP-05.

\section{Footnote}

Conflicts of Interest: All authors have completed the ICMJE uniform disclosure form (available at https://dx.doi. org/10.21037/tp-21-239). The authors have no conflicts of interest to declare.

Etbical Statement: The authors are accountable for all aspects of the work in ensuring that questions related to the accuracy or integrity of any part of the work are appropriately investigated and resolved. The study will be conducted in accordance with the Declaration of Helsinki (as revised in 2013). The study was approved by ethics board of the Chongqing Medical University Affiliated Children's Hospital (No.: 2020-217) and informed consent will be taken from all individual participants once the study is initiated.

Open Access Statement: This is an Open Access article distributed in accordance with the Creative Commons Attribution-NonCommercial-NoDerivs 4.0 International License (CC BY-NC-ND 4.0), which permits the noncommercial replication and distribution of the article with the strict proviso that no changes or edits are made and the original work is properly cited (including links to both the formal publication through the relevant DOI and the license). See: https://creativecommons.org/licenses/by-nc-nd/4.0/.

\section{References}

1. Akinbami L; Centers for Disease Control and Prevention National Center for Health Statistics. The state of childhood asthma, United States, 1980-2005. Adv Data 2006;(381):1-24.

2. Li X, Song P, Zhu Y, et al. The disease burden of childhood asthma in China: a systematic review and meta- analysis. J Glob Health 2020;10:010801.

3. Taussig LM, Wright AL, Holberg CJ, et al. Tucson Children's Respiratory Study: 1980 to present. J Allergy Clin Immunol 2003;111:661-75; quiz 676.

4. De Sario M, Di Domenicantonio R, Corbo G, et al. Characteristics of early transient, persistent, and late onset wheezers at 9 to 11 years of age. J Asthma 2006;43:633-8.

5. Reddel HK, Busse WW, Pedersen S, et al. Should recommendations about starting inhaled corticosteroid treatment for mild asthma be based on symptom frequency: a post-hoc efficacy analysis of the START study. Lancet 2017;389:157-66.

6. Bao Y, Chen Z, Liu E, et al. Risk Factors in Preschool Children for Predicting Asthma During the Preschool Age and the Early School Age: a Systematic Review and Meta-Analysis. Curr Allergy Asthma Rep 2017;17:85.

7. Smit HA, Pinart M, Antó JM, et al. Childhood asthma prediction models: a systematic review. Lancet Respir Med 2015;3:973-84.

8. Kothalawala DM, Kadalayil L, Weiss VBN, et al. Prediction models for childhood asthma: A systematic review. Pediatr Allergy Immunol 2020;31:616-27.

9. Papi A, Brightling C, Pedersen SE, et al. Asthma. Lancet 2018;391:783-800.

10. Turner S, Fielding S, Mullane D, et al. A longitudinal study of lung function from 1 month to 18 years of age. Thorax 2014;69:1015-20.

11. Stern DA, Morgan WJ, Wright AL, et al. Poor airway function in early infancy and lung function by age 22 years: a non-selective longitudinal cohort study. Lancet 2007;370:758-64.

12. Håland G, Carlsen KC, Sandvik L, et al. Reduced lung function at birth and the risk of asthma at 10 years of age. N Engl J Med 2006;355:1682-9.

13. van der Wiel E, ten Hacken NH, Postma DS, et al. Smallairways dysfunction associates with respiratory symptoms and clinical features of asthma: a systematic review. J Allergy Clin Immunol 2013;131:646-57.

14. Lipworth B, Manoharan A, Anderson W. Unlocking the quiet zone: the small airway asthma phenotype. Lancet Respir Med 2014;2:497-506.

15. Balzar S, Wenzel SE, Chu HW. Transbronchial biopsy as a tool to evaluate small airways in asthma. Eur Respir J 2002;20:254-9.

16. Nihlberg K, Andersson-Sjöland A, Tufvesson E, et al. Altered matrix production in the distal airways of 
individuals with asthma. Thorax 2010;65:670-6.

17. Knihtilä H, Kotaniemi-Syrjänen A, Mäkelä MJ, et al. Preschool oscillometry and lung function at adolescence in asthmatic children. Pediatr Pulmonol 2015;50:1205-13.

18. Galant SP, Fregeau W, Pabelonio N, et al. Standardized IOS Reference Values Define Peripheral Airway Impairment-Associated Uncontrolled Asthma Risk Across Ethnicity in Children. J Allergy Clin Immunol Pract 2020;8:2698-706.

19. Bjermer L. The role of small airway disease in asthma. Curr Opin Pulm Med 2014;20:23-30.

20. Korevaar DA, Westerhof GA, Wang J, et al. Diagnostic accuracy of minimally invasive markers for detection of airway eosinophilia in asthma: a systematic review and meta-analysis. Lancet Respir Med 2015;3:290-300.

21. Chang D, Yao W, Tiller CJ, et al. Exhaled nitric oxide during infancy as a risk factor for asthma and airway hyperreactivity. Eur Respir J 2015;45:98-106.

22. Lezmi G, Deschildre A, Abou Taam R, et al. Remodelling and inflammation in preschoolers with severe recurrent wheeze and asthma outcome at school age. Clin Exp Allergy 2018;48:806-13.

23. Kropski JA, Fremont RD, Calfee CS, et al. Clara cell protein (CC16), a marker of lung epithelial injury, is decreased in plasma and pulmonary edema fluid from patients with acute lung injury. Chest 2009;135:1440-7.

24. Guerra S, Vasquez MM, Spangenberg A, et al. Club cell secretory protein in serum and bronchoalveolar lavage of patients with asthma. J Allergy Clin Immunol 2016;138:932-934.e1.

25. Zeng J, Chen Z, Hu Y, et al. Asthma control in preschool children with small airway function as measured by IOS and fractional exhaled nitric oxide. Respir Med 2018;145:8-13.

26. Ducharme FM, Dell SD, Radhakrishnan D, et al. Diagnosis and management of asthma in preschoolers: A Canadian Thoracic Society and Canadian Paediatric Society position paper. Paediatr Child Health 2015;20:353-71.

27. Global initiative for asthma. Global strategy for asthma management and prevention, 2020. Available online: www. ginasthma.org (Accessed 2 November 2020).

28. Liu AH, Zeiger R, Sorkness C, et al. Development and cross-sectional validation of the Childhood Asthma Control Test. J Allergy Clin Immunol 2007;119:817-25.

29. Wu J, Yang Y, Zhang J, et al. Research into the application of asthma control test in children. Chin J Practical Pediatr 2011;26:256-9.
30. TRACK: Test for Respiratory and Asthma Control in Kids. Available online: http://www.asthmatracktest.com/ pdf/Track_Tearpad.pdf (Accessed 16 August 2020).

31. Murphy KR, Zeiger RS, Kosinski M, et al. Test for respiratory and asthma control in kids (TRACK): a caregiver-completed questionnaire for preschool-aged children. J Allergy Clin Immunol 2009;123:833-9.e9.

32. Lajunen K, Kalliola S, Kotaniemi-Syrjänen A, et al. Abnormal lung function at preschool age asthma in adolescence? Ann Allergy Asthma Immunol 2018;120:520-6.

33. Beydon N, Davis SD, Lombardi E, et al. An official American Thoracic Society/European Respiratory Society statement: pulmonary function testing in preschool children. Am J Respir Crit Care Med 2007;175:1304-45.

34. Oostveen E, MacLeod D, Lorino H, et al. The forced oscillation technique in clinical practice: methodology, recommendations and future developments. Eur Respir J 2003;22:1026-41.

35. Bickel S, Popler J, Lesnick B, et al. Impulse oscillometry: interpretation and practical applications. Chest 2014;146:841-7.

36. Malmberg LP, Pelkonen A, Poussa T, et al. Determinants of respiratory system input impedance and bronchodilator response in healthy Finnish preschool children. Clin Physiol Funct Imaging 2002;22:64-71.

37. Miller MR, Hankinson J, Brusasco V, et al. Standardisation of spirometry. Eur Respir J 2005;26:319-38.

38. Baraldi E, de Jongste JC; European Respiratory Society/ American Thoracic Society (ERS/ATS) Task Force. Measurement of exhaled nitric oxide in children, 2001. Eur Respir J 2002;20:223-37.

39. Warke TJ, Fitch PS, Brown V, et al. Exhaled nitric oxide correlates with airway eosinophils in childhood asthma. Thorax 2002;57:383-7.

40. Knihtilä H, Kotaniemi-Syrjänen A, Pelkonen AS, et al. Small airway function in children with mild to moderate asthmatic symptoms. Ann Allergy Asthma Immunol 2018;121:451-7.

41. Harris PA, Taylor R, Minor BL, et al. The REDCap consortium: Building an international community of software platform partners. J Biomed Inform 2019;95:103208.

42. EMEA. Guideline on missing data in confirmatory clinical trials. Available online: https://www.ema.europa. eu/en/documents/scientific-guideline/guideline-missingdata-confirmatory-clinical-trials_en.pdf (Accessed 16 August 2020). 
43. Konstantinos Katsoulis K, Kostikas K, Kontakiotis T. Techniques for assessing small airways function: Possible applications in asthma and COPD. Respir Med 2016;119:e2-9.

Cite this article as: Li Q, Zhou Q, Li Y, Liu E, Fu Z, Luo J, Liu S, Liu F, Chen Y, Luo Z. The predictive role of small airway dysfunction and airway inflammation biomarkers for asthma in preschool and school-age children: a study protocol for a prospective cohort study. Transl Pediatr 2021;10(10):26302638. doi: 10.21037/tp-21-239
44. Anderson WJ, Zajda E, Lipworth BJ. Are we overlooking persistent small airways dysfunction in communitymanaged asthma? Ann Allergy Asthma Immunol 2012;109:185-189.e2. 